\title{
Relations between loss angles in isotropic linear viscoelastic materials *)
}

\author{
H. A. Waterman
}

With 16 figures and 1 table

\section{Introduction}

For the description of the mechanical behaviour of linear viscoelastic materials a number of characteristic material properties is in use. So the relaxation functions in shear, $J(t)$, in extension, $D(t)$, and in compression, $B(t)$, describe the response of the material to a stepfunction $H(t)$ in the corresponding stress. It can be shown theoretically that the relaxation functions are monotonically decreasing functions of time, whereas the retardation functions are monotonically increasing functions of time. It is obvious that Poisson's ratio of a viscoelastic material too is a function of time, so one could define a relaxation Poisson's ratio, c.q. a retardation Poisson's ratio. It cannot be proved, however, that these functions are monotonically decreasing, resp. increasing with time. In case of harmonic deformations a complex Poisson's ratio $v^{*} \equiv v^{\prime}+i v^{\prime \prime}$ can be defined. But also here it cannot be proved that complex Poisson's ratio shows either the characteristics of a modulus or of a compliance.

The variation of complex Poisson's ratio has been studied theoretically by Gottenberg and Christensen (1) for the special cases where either one or both of $G^{\prime}$ and $G^{\prime \prime}$ assume finite non zero values and $\kappa^{\prime}$ or $\kappa^{\prime \prime}$ tend to zero. It turned out that $v^{\prime}$ is varying between -1 and 0.5 , while $v^{\prime \prime}$ may take positive or negative values. In a similar study Rigbi (2) discussed the special cases where either $v^{\prime \prime}=0$ or $\kappa^{\prime \prime}=0$.

An experimental study of the complex moduli and Poisson's ratio of several polymers was performed by the present author $(3,4)$, from which it appeared that $v^{\prime}>0$ and $v^{\prime \prime}<0$. Using

*) Part of this study was performed while the author was an employee of TNO, Delft. 335 these constraints on $v^{\prime}$ and $v^{\prime \prime}$ Theocaris (5) deduced the sequence of the magnitudes of several loss angles. In this paper the same goal will be achieved along a different line and it will be shown that some propositions deduced by Theocaris (5) are either incorrect or only correct for a more severe constraint on $v^{\prime}$. In accordance with the former authors we shall confine ourselves to homogeneous, isotropic materials, so two complex moduli (f. i. complex shear modulus $G^{*}(\omega)$ and complex bulk modulus $\kappa^{*}(\omega)$ ) suffice for a complete description of the viscoelastic behaviour. The only requirements regarding the form of a complex modulus is that both its real and imaginary part is non negative. This is a consequence of the requirements of non negative energy storage and non negative energy dissipation (6). The complex quantities to be discussed are:

$$
\begin{aligned}
G^{*}(i \omega) & =G^{\prime}(\omega)+i G^{\prime \prime}(\omega)-\text { shear modulus } \\
\kappa^{*}(i \omega) & =\kappa^{\prime}(\omega)+i \kappa^{\prime \prime}(\omega)-\text { bulk modulus } \\
E^{*}(i \omega) & =E^{\prime}(\omega)+i E^{\prime \prime}(\omega)-\text { Young's modulus } \\
\nu^{*}(i \omega) & =\nu^{\prime}(\omega)+i \nu^{\prime \prime}(\omega)-\text { Poisson's ratio } \\
\lambda^{*}(i \omega) & =\lambda^{\prime}(\omega)+i \lambda^{\prime \prime}(\omega)-\text { second } \\
L^{*}(i \omega) & =\lambda^{*}(i \omega)+2 G^{*}(i \omega) \\
& =L^{\prime}(\omega)+i L^{\prime}(\omega)-\text { longitudinal modulus }
\end{aligned}
$$

\section{Calculations}

The relations between the various complex functions for an isotropic linear viscoelastic material are compiled in table 1.

**) By its definition the second Lamé function has not the properties of a modulus, i.e. both the real and imaginary part of $\lambda^{*}(i \omega)$ can be negative. The same applies to Poisson's ratio. 


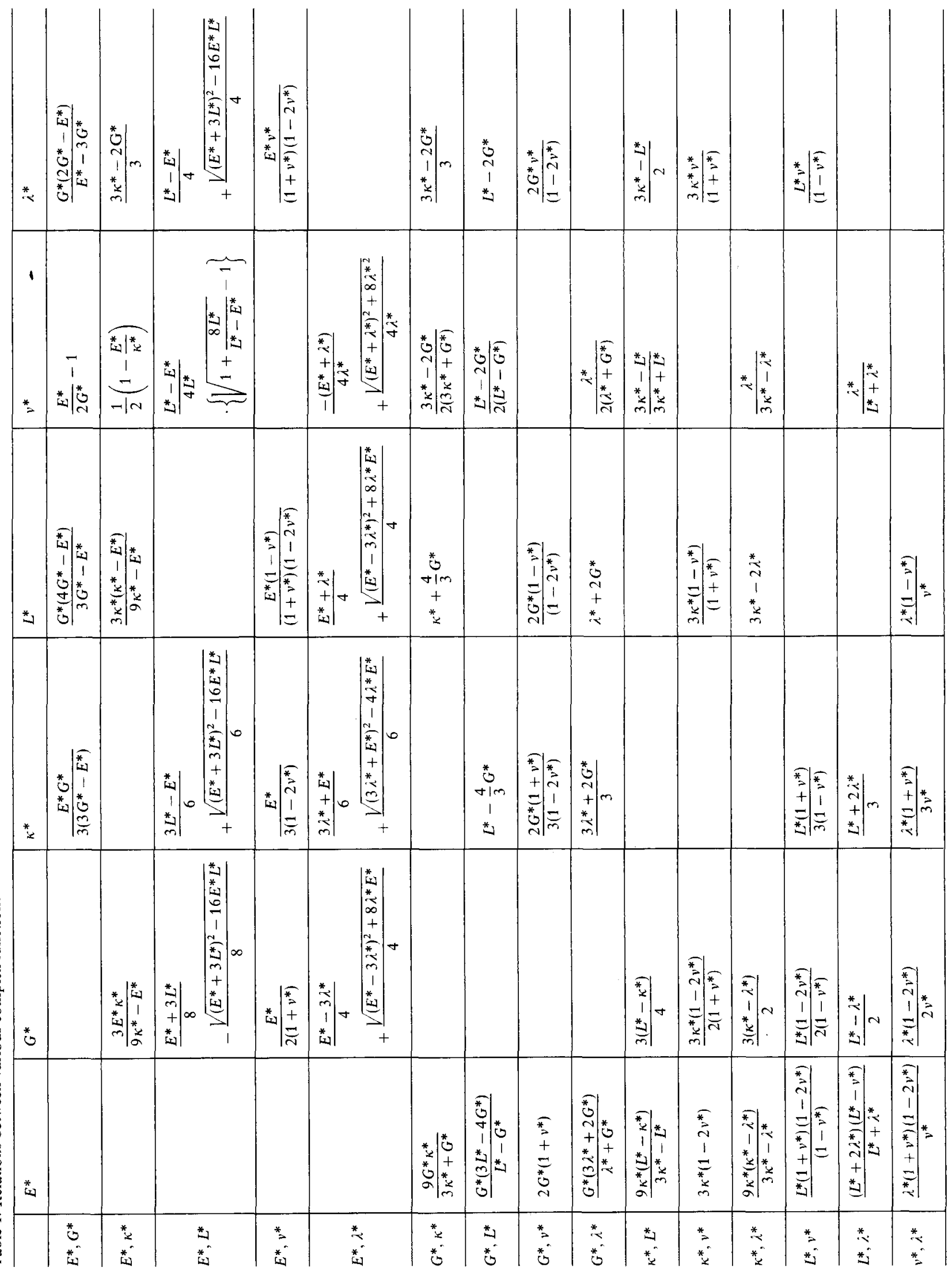


From table 1 we read:

$1-2 v^{*}=\frac{E^{*}}{3 \kappa^{*}}$.

Equating the real and imaginary parts of both sides of eq. [1] and dividing one of the resulting equations by the other one leads to:

$\beta \equiv \frac{-2 v^{\prime \prime}}{1-2 v^{\prime}}=\frac{\kappa^{\prime} E^{\prime \prime}-E^{\prime} \kappa^{\prime \prime}}{\kappa^{\prime} E^{\prime}+\kappa^{\prime \prime} E^{\prime \prime}}=\tan \left(\delta_{E}-\delta_{\kappa}\right)$

with

$\tan \delta_{\kappa} \equiv \frac{\kappa^{\prime \prime}}{\kappa^{\prime}}$ etc.

For a fixed value of $\beta$ eq. [2] represents in the $v^{\prime}, v^{\prime \prime}$ plane a line through $\left[\frac{1}{2} ; 0\right]$ with the slope $\beta=\tan \left(\delta_{E}-\delta_{\kappa}\right)$, see fig. 1. This line is the locus of $v^{*}$ for this particular value of $\left(\delta_{E}-\delta_{\kappa}\right)$. From [2] it follows further:

$v^{\prime \prime}=0 ; v^{\prime} \neq \frac{1}{2} \rightarrow \tan \delta_{E}=\tan \delta_{\kappa}$.

In the same way from

$1+v^{*}=\frac{E^{*}}{2 G^{*}}$

it follows

$\alpha \equiv \frac{-v^{\prime \prime}}{1+v^{\prime}}=\tan \left(\delta_{G}-\delta_{E}\right)$.

Eqs. [2] and [5] were derived before by Schwarzl and Burgers (7) in a somewhat different way.

For a fixed value of $\alpha$ eq. [5] represents in the $v^{\prime}, v^{\prime \prime}$ plane a line through $[-1 ; 0]$ with the slope $-\tan \left(\delta_{G}-\delta_{E}\right)$. This line is the locus of $v^{*}$ for this particular value of $\left(\delta_{G}-\delta_{E}\right)$, see fig. 1 . Obviously $v^{*}$ is determined by two differences between loss angles. From [5] it further follows:

$v^{\prime \prime}=0 ; v^{\prime} \neq-1 \rightarrow \tan \delta_{G}=\tan \delta_{E}$.

From [3] and [6] it is concluded:

$\left.\begin{array}{l}v^{\prime \prime}=0 \\ v^{\prime} \neq \frac{1}{2} ; v^{\prime} \neq-1\end{array}\right\} \rightarrow \tan \delta_{\kappa}=\tan \delta_{G}=\tan \delta_{E}$.

In an earlier paper Rigbi (8) concluded that $v^{\prime \prime}=0$ if, and only if $E^{\prime \prime}=G^{\prime \prime}=0$, which means: $\tan \delta_{E}=\tan \delta_{G}=0$. From inspection of fig. 1 (from which [7] is revealed directly) it is seen that the criterion of Rigbi (3) is only a special case of [7].

From fig. 1 it follows, using a simple geometric theorem, that for a fixed value of $\left(\delta_{G}-\delta_{\kappa}\right)$ (all triangles $\mathrm{ABC}$ then have the same vertical angle) the locus of $\nu^{*}$ is a circle passing through $\left[\frac{1}{2} ; 0\right]$ and $[-1 ; 0]$, intersecting the positive $v^{\prime}$ axis at the slope $\tan \left(\delta_{G}-\delta_{\kappa}\right)$. Analytically this circle can be found from the relation

$\kappa^{*}=G^{*} \frac{2\left(1+v^{*}\right)}{3\left(1-2 v^{*}\right)}$.

Equating the real and imaginary parts of both sides of eq. [8] and dividing one of the resulting equations by the other one leads to:

$\tan \delta_{\kappa}=\frac{\left\{1-v^{\prime}-2\left(v^{\prime}\right)^{2}-2\left(v^{\prime \prime}\right)^{2}\right\} \tan \delta_{G}-3 v^{\prime \prime}}{\left\{1-v^{\prime}-2\left(v^{\prime}\right)^{2}-2\left(v^{\prime \prime}\right)^{2}\right\}+3 v^{\prime \prime} \tan \delta_{G}}$,

from which follows:

$\left\{1-v^{\prime}-2\left(v^{\prime}\right)^{2}-2\left(v^{\prime \prime}\right)^{2}\right\} \tan \left(\delta_{G}-\delta_{\kappa}\right)-3 v^{\prime \prime}=0$.

By completing the squares eqs. [10] can be written as

$$
\begin{aligned}
\left(v^{\prime}+\frac{1}{4}\right)^{2} & +\left\{v^{\prime \prime}-\frac{3}{4 \tan \left(\delta_{G}-\delta_{\kappa}\right)}\right\}^{2} \\
& =\left\{\frac{3}{4 \sin \left(\delta_{G}-\delta_{\kappa}\right)}\right\}^{2},
\end{aligned}
$$

which represents the circle mentioned.

Now from $G^{\prime} \geq 0, G^{\prime \prime} \geq 0$ etc. it follows:

$0 \leq \delta_{\text {G.K.E }} \leq \pi / 2$,

so $\left|\delta_{G}-\delta_{\kappa}\right| \leq \pi / 2$. From inspection of fig. 1 it is then learned that $-1 \leq v^{\prime} \leq \frac{1}{2}$, which is the same range found from the theory of linear elasticity.

For $\delta_{\kappa}=0$ the circle represented by [11] intersects the positive $v^{\prime}$ axis at the slope $\tan \delta_{G}$. The latter circle was mentioned before by Gottenberg and Christensen (1). If moreover $\delta_{G}=\pi / 2$, the centre of the circle is located on the $v^{\prime}$ axis (see fig. 2) and then the radius of the circle equals the maximum value of $\left|v^{\prime \prime}\right|$. A similar situation arises for $\delta_{G}=0$ and $\delta_{\kappa}=\pi / 2$. From fig. 2 it is seen

$-\frac{3}{4} \leq v^{\prime \prime} \leq \frac{3}{4}$.

For $\left|\delta_{G}-\delta_{\kappa}\right| \leq 1.25$ and $\left|\delta_{G}-\delta_{\kappa}\right| \leq \pi / 2 v^{*}$ is located in the shaded areas of fig. 1 and fig. 2 respectively.

In studying the mechanical behaviour of viscoelastic materials, besides techniques for measuring $E^{*}$ and $G^{*}$, wave propagation methods 


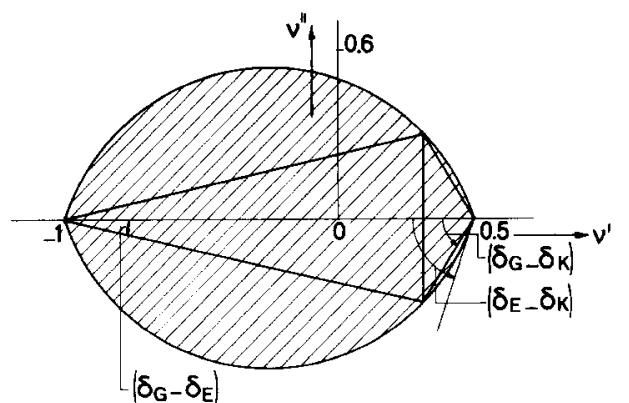

Fig. 1. Locus of complex Poisson's ratio $v^{*}$ for fixed values of $\left|\delta_{G}-\delta_{E}\right|,\left|\delta_{E}-\delta_{\kappa}\right|$ and $\left|\delta_{G}-\delta_{\kappa}\right|$ respectively

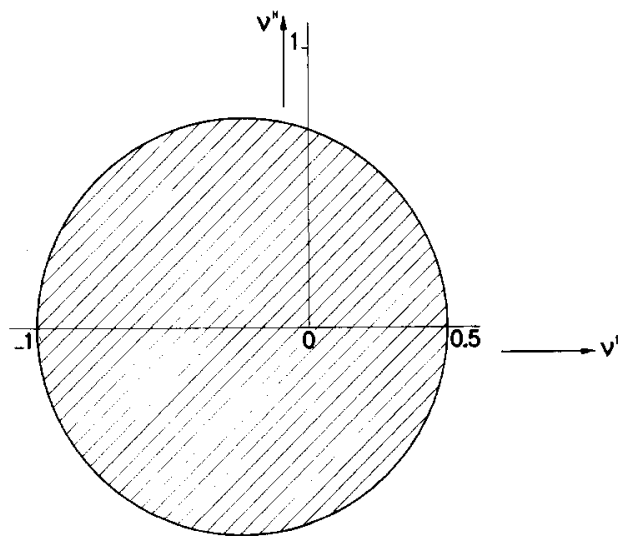

Fig. 2. Locus of complex Poisson's ratio $v^{*}$ for $\left|\delta_{G}-\delta_{\kappa}\right|=\pi / 2$

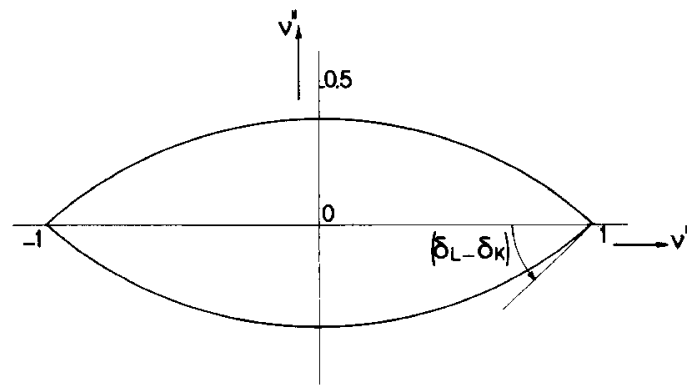

Fig. 3. Locus of complex Poisson's ratio $v^{*}$ for a fixed value of $\left|\delta_{L}-\delta_{\kappa}\right|$

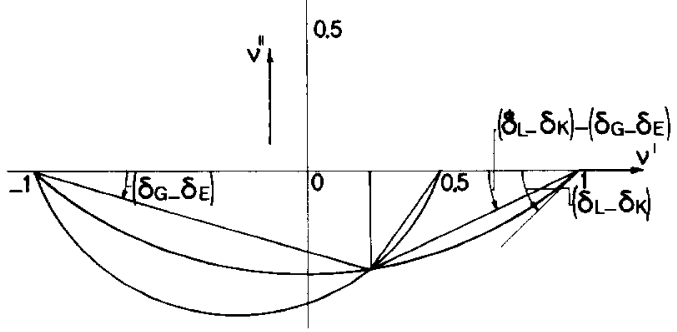

Fig. 4. Combination of figs. 1 and 3 for $\delta_{G}>\delta_{E}$ are in use from which both $G^{*}$ and $L^{*}$ are determined. It is of some interest therefore to include $L^{*}$ in our examinations. From table 1 we read:

$\kappa^{*}=L^{*} \frac{\left(1+v^{*}\right)}{3\left(1-v^{*}\right)}$.

From [13] it is found that for a fixed value of $\left(\delta_{L}-\delta_{\kappa}\right)$ the locus of $v^{*}$ is a circle the centre of which is located on the $v^{\prime \prime}$ axis. This circle intersects the positive $v^{\prime}$ axis at the slope $\tan \left(\delta_{L}-\delta_{\kappa}\right)$ in $[1 ; 0]$, see fig. 3. In fig. 4 we combined figs. 1 and 3 for the case $\delta_{G}>\delta_{E}$. From fig. 4 the following relation is found from simple geometrical arguments:

$\gamma \equiv \frac{-v^{\prime \prime}}{1-v^{\prime}}=\tan \left\{\left(\delta_{L}-\delta_{\kappa}\right)-\left(\delta_{G}-\delta_{E}\right)\right\}$.

This relation is of an analogous form as [2] and [5], but it has not yet been mentioned in literature. It can be found analytically from

$\frac{L^{*} / \kappa^{*}}{G^{*} / E^{*}}=6\left(1-v^{*}\right)$,

see table 1 .

\section{Relationships between loss tangents}

From fig. 1 it follows that

$v^{\prime \prime} \lessgtr 0 \leftrightarrow \tan \delta_{G} \gtrless \tan \delta_{E} \gtrless \tan \delta_{\kappa}$.

We can wonder now whether the position of $\tan \delta_{L}$ in this row can be located. From [14], [15] and [12] we conclude

$v^{\prime \prime} \lessgtr 0 \leftrightarrow \tan \delta_{L} \gtrless \tan \delta_{\kappa}$.

Further we prove:

$v^{\prime \prime} \gtrless 0 \leftrightarrow \tan \delta_{L} \lessgtr \tan \delta_{G}$

or

$v^{\prime \prime} \lessgtr 0 \leftrightarrow \frac{\kappa^{\prime \prime}+\frac{4}{3} G^{\prime \prime}}{\kappa^{\prime}+\frac{4}{3} G^{\prime}} \lessgtr \frac{G^{\prime \prime}}{G^{\prime}}$

which reduces to

$v^{\prime \prime} \lessgtr 0 \leftrightarrow \frac{\kappa^{\prime \prime}}{\kappa^{\prime}} \lessgtr \frac{G^{\prime \prime}}{G^{\prime}}$,

and this indeed follows from [15].

Further we try to find the position of $\tan \delta_{L}$ with respect to $\tan \delta_{E}$. It is seen from table 1 that $\operatorname{Im} . L^{*} / E^{*}$, the imaginary part of $L^{*} / E^{*}$, is: 


$$
\begin{aligned}
\operatorname{Im} . L^{*} / E^{*} & =\frac{L^{\prime} E^{\prime}\left(\tan \delta_{L}-\tan \delta_{E}\right)}{\left(E^{\prime}\right)^{2}+\left(E^{\prime \prime}\right)^{2}} \\
& =\operatorname{Im} \cdot \frac{1-v^{*}}{\left(1+v^{*}\right)\left(1-2 v^{*}\right)} \\
= & \frac{v^{\prime \prime}\left\{-2\left(v^{\prime}\right)^{2}-2\left(v^{\prime \prime}\right)^{2}+4 v^{\prime}\right\}}{\left\{1-v^{\prime}-2\left(v^{\prime}\right)^{2}+2\left(v^{\prime \prime}\right)^{2}\right\}^{2}+\left\{v^{\prime \prime}+4 v^{\prime} v^{\prime \prime}\right\}^{2}}
\end{aligned}
$$

Excluding $\left[\frac{1}{2} ; 0\right]$ the denominator of $[18]$ is always positive. The numerator of [18] can be written in the form

$-2 v^{\prime \prime}\left\{\left(v^{\prime}-1\right)^{2}+\left(v^{\prime \prime}\right)^{2}-1\right\}$.

From [18] it can be concluded therefore:

$\left.\begin{array}{l}v^{\prime \prime} \lessgtr 0 \\ \left(v^{\prime}-1\right)^{2}+\left(v^{\prime \prime}\right)^{2}<1\end{array}\right\} \rightarrow \tan \delta_{E} \gtrless \tan \delta_{L}$.

In the $v^{\prime}, v^{\prime \prime}$ plane the equation $\left(v^{\prime}-1\right)^{2}+$ $\left(v^{\prime \prime}\right)^{2}=1$ is represented by the dotted circle in fig. 5. From this figure it is seen that the value of $v^{\prime}$ for which $\tan \delta_{E}=\tan \delta_{L}$ depends on $\left|\delta_{G}-\delta_{\kappa}\right|$. It is evident, however, that for $v^{\prime}>0.2$ we have

$\left.\begin{array}{l}v^{\prime \prime} \lessgtr 0 \\ v^{\prime}>0.2\end{array}\right\} \rightarrow \tan \delta_{E} \gtrless \tan \delta_{L}$.

From fig. 5 it is also seen:

$\left.\begin{array}{l}v^{\prime \prime} \lessgtr 0 \\ v^{\prime}<0\end{array}\right\} \rightarrow \tan \delta_{E} \lessgtr \tan \delta_{L}$.

Let us assume $v^{\prime}>0.2$; then from [15], [16] and $[20]$ it follows:

$\left.\begin{array}{l}v^{\prime \prime} \lessgtr 0 \\ v^{\prime}>0.2\end{array}\right\} \rightarrow \tan \delta_{G} \gtrless \tan \delta_{E} \gtrless \tan \delta_{L} \gtrless \tan \delta_{\kappa}$.

A proposition similar to $[22]$ is given by Theocaris (5), viz.

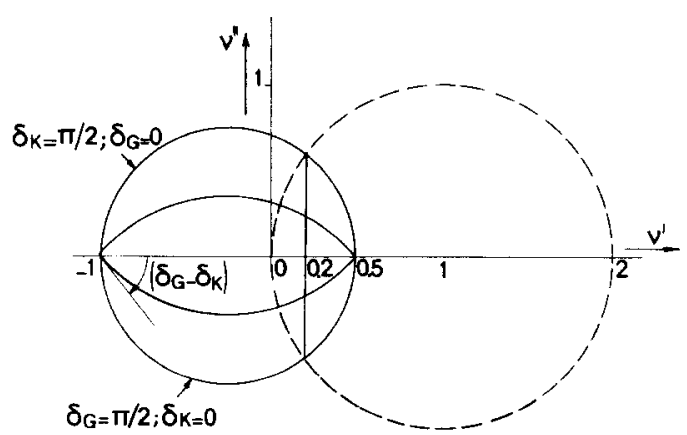

Fig. 5. Illustration to the inequalities [19] and [20]

$$
\left.\begin{array}{c}
v^{\prime \prime}<0 \\
v^{\prime}>0
\end{array}\right\}>\tan \delta_{G}>\tan \delta_{E}>\tan \delta_{L}
$$

where

$\tan \delta_{v} \equiv \frac{\left|v^{\prime \prime}\right|}{v^{\prime}}$

In deriving the proposition

$\left.\begin{array}{l}v^{\prime \prime}<0 \\ v^{\prime}>0\end{array}\right\} \rightarrow \tan \delta_{E}>\tan \delta_{L}$

Theocaris showed:

$v^{\prime}>0$

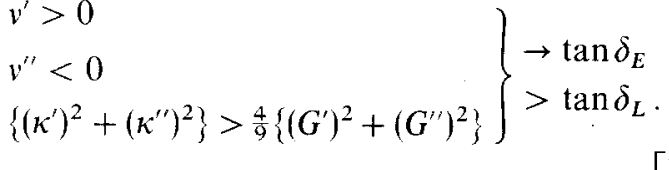

The proposition

$\left\{\left(\kappa^{\prime}\right)^{2}+\left(\kappa^{\prime \prime}\right)^{2}\right\}>\frac{4}{9}\left\{\left(G^{\prime}\right)^{2}+\left(G^{\prime \prime}\right)^{2}\right\}$

is, without proof, claimed to hold rigorously by Theocaris (5). It is easily shown, however, that $[25]$ is only valid with some restriction. From table 1 it follows:

$\left|\kappa^{*}\right|=\frac{2}{3}\left|G^{*}\right| \cdot \frac{\left|1+v^{*}\right|}{\left|1-2 v^{*}\right|}$

or

$$
\begin{aligned}
\left\{\left(\kappa^{\prime}\right)^{2}+\left(\kappa^{\prime \prime}\right)^{2}\right\}= & \frac{4}{9}\left\{\left(G^{\prime}\right)^{2}+\left(G^{\prime \prime}\right)^{2}\right\} \\
& \cdot\left\{\frac{\left(1+v^{\prime}\right)^{2}+\left(v^{\prime \prime}\right)^{2}}{\left(1-2 v^{\prime}\right)^{2}+4\left(v^{\prime \prime}\right)^{2}}\right\} .
\end{aligned}
$$

So for [25] to be valid the following inequality must hold:

$\left(1-2 v^{\prime}\right)^{2}+4\left(v^{\prime \prime}\right)^{2}<\left(1+v^{\prime}\right)^{2}+\left(v^{\prime \prime}\right)^{2}$

or

$\left(v^{\prime}-1\right)^{2}+\left(v^{\prime \prime}\right)^{2}<1$.

With this restriction [24] renders into [19].

As for the proposition

$\left.\begin{array}{l}v^{\prime \prime}<0 \\ v^{\prime}>0\end{array}\right\} \tan \delta_{\kappa}>\tan \delta_{v}$

Theocaris (5) showed

$$
\left.\begin{array}{rl}
v^{\prime} & >0 \\
v^{\prime \prime} & <0 \\
3 \kappa^{\prime \prime} & >E^{\prime \prime}
\end{array}\right\} \rightarrow \tan \delta_{\kappa}>\tan \delta_{v} .
$$


The proposition $3 \kappa^{\prime \prime}>E^{\prime \prime}$ is deduced by this author from an argument I could not understand. Now from eq. [1] it is found:

$3 \kappa^{\prime \prime}=E^{\prime \prime} \frac{\left(1-2 v^{\prime}\right)+2 v^{\prime \prime} / \tan \delta_{E}}{\left(1-2 v^{\prime}\right)^{2}+4\left(v^{\prime \prime}\right)^{2}}$,

from which it follows that $3 \kappa^{\prime \prime}>E^{\prime \prime}$ if

$\left(1-2 v^{\prime}\right)+2 v^{\prime \prime} / \tan \delta_{E}>\left(1-2 v^{\prime}\right)^{2}+4\left(v^{\prime \prime}\right)^{2}$,

which after some manipulation leads to

$\left(2 v^{\prime}-\frac{1}{2}\right)^{2}+\left(2 v^{\prime \prime}-\frac{1}{2 \tan \delta_{E}}\right)^{2}<\left(\frac{1}{2 \sin \delta_{E}}\right)^{2}$.

Eq. [28], with $<$ substituted by $=$, represents a circle passing through $[0 ; 0]$ and $\left[\frac{1}{2} ; 0\right]$. For points within this circle $3 \kappa^{\prime \prime}>E^{\prime \prime}$ and therefore $\tan \delta_{\kappa}>\tan \delta_{y}$, while for points outside this circle $\tan \delta_{v}>\tan \delta_{\kappa}$.

Another bound that could be established is

$$
\left.\begin{array}{rl}
v^{\prime \prime} & <0 \\
v^{\prime} & >0 \\
E^{\prime \prime} & >2 G^{\prime \prime}
\end{array}\right\} \rightarrow \tan \delta_{G}>\tan \delta_{v} .
$$

\section{Suppose}

$\frac{G^{\prime \prime}}{G^{\prime}}>\frac{\left|v^{\prime \prime}\right|}{v^{\prime}}$

[30]

now from [4] it follows:

$v^{\prime}=\frac{E^{\prime} G^{\prime}+E^{\prime \prime} G^{\prime \prime}}{2\left\{\left(G^{\prime}\right)^{2}+\left(G^{\prime \prime}\right)^{2}\right\}}-1$

$\left.\left|v^{\prime \prime}\right|=-v^{\prime \prime}=\frac{E^{\prime} G^{\prime \prime}-G^{\prime} E^{\prime \prime}}{2\left\{\left(G^{\prime}\right)^{2}+\left(G^{\prime \prime}\right)^{2}\right\}}\right\}$

Introducing [31] into [30] yields :

$E^{\prime \prime}>2 G^{\prime \prime}$.
From [4] it also follows:

$E^{\prime \prime}=2 G^{\prime \prime} \frac{\left(1+v^{\prime}\right)^{2}+\left(v^{\prime \prime}\right)^{2}}{1+v^{\prime}-v^{\prime \prime} / \tan \delta_{E}}$.

So $E^{\prime \prime}>2 G^{\prime \prime}$ if

$\left(1+v^{\prime}\right)^{2}+\left(v^{\prime \prime}\right)^{2}>1+v^{\prime}-v^{\prime \prime} / \tan \delta_{E}$,

which can be rewritten as

$\left(v^{\prime}+\frac{1}{2}\right)^{2}+\left(v^{\prime \prime}+\frac{1}{2 \tan \delta_{E}}\right)^{2}>\left(\frac{1}{2 \sin \delta_{E}}\right)^{2}$.

Eq. [34], with $>$ substituted by $=$, represents a circle passing through $[-1,0]$ and $[0,0]$, intersecting the positive $v^{\prime}$ axis at the latter point with the slope $-\tan \delta_{E}$, see fig. 6 . On this circle $\delta_{G}=\delta_{v}$, as is readily confirmed by simple geometric arguments. For points outside this circle $\delta_{G}>\delta_{v}$. In fig. 7 we combined figs. 5 and 6 . Also shown in this figure is the circle represented by [28]. It is clear from this figure that for a given value of $\left(\delta_{G}-\delta_{\kappa}\right)$ for which $\left(\delta_{G}-\delta_{\kappa}\right)<\delta_{E}$

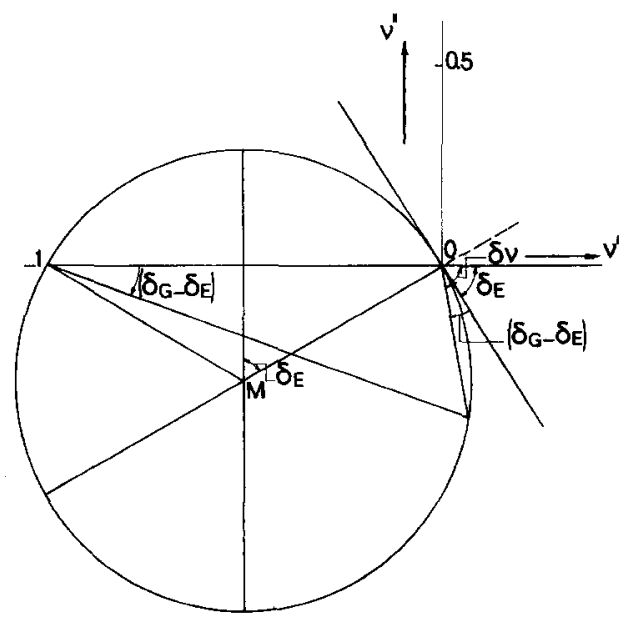

Fig. 6. Graphic representation of [34] (with $>$ substituted by $=$ ) to illustrate the inequality [29]

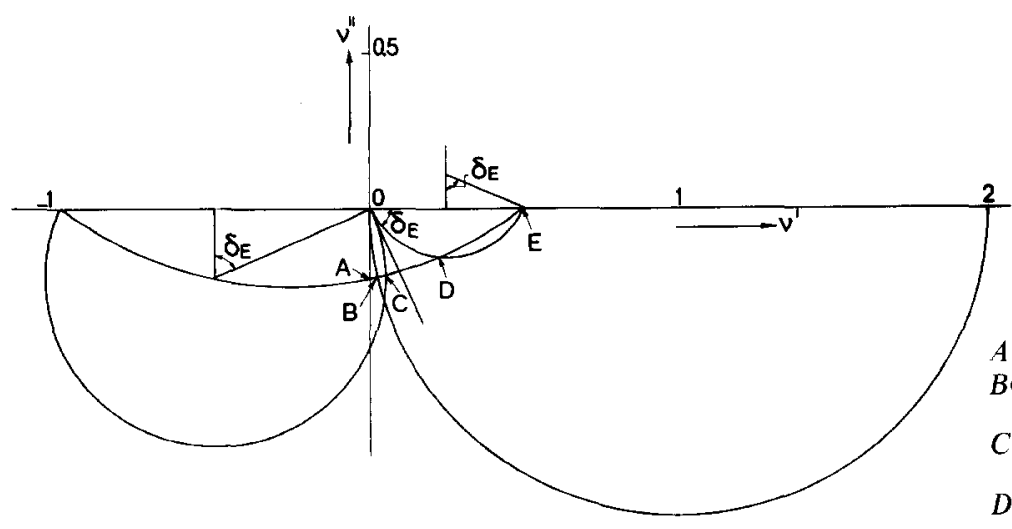

Fig. 7. Combination of figs. 5 and 6 , illustrating the various regions for which different inequalities are valid. In this figure we chose $\delta_{G}-\delta_{\kappa}<\delta_{E}$

$A B: \tan \delta_{\mathrm{v}}>\tan \delta_{G}>\tan \delta_{L}>\tan \delta_{E}>\tan \delta_{\kappa}$ $B C: \tan \delta_{v}>\tan \delta_{G}>\tan \delta_{E}>\tan \delta_{L}>\tan \delta_{\kappa}$ $C D:\left\{\begin{array}{l}\tan \delta_{G}>\tan \delta_{E}>\tan \delta_{L}>\tan \delta_{\kappa} \\ \tan \delta_{G}>\tan \delta_{v}>\tan \delta_{\kappa}\end{array}\right.$

$D E: \tan \delta_{G}>\tan \delta_{E}>\tan \delta_{L}>\tan \delta_{\kappa}>\tan \delta_{v}$ 
four regions can be distinguished as illustrated in fig. 7.

A similar situation arises for $v^{\prime \prime}>0, v^{\prime}<0$; i. e. a relation analogous to [22] can be derived. The conditions $v^{\prime \prime}>0, v^{\prime}<0$ have no practical value, however, as will be shown later on in this paper. For the other combinations, viz. $v^{\prime \prime}<0, v^{\prime}<0$ and $v^{\prime \prime}>0, v^{\prime}>0$, no simple bounds can be established.

\section{On the second Lamé function}

As mentioned before $\lambda^{*}$ is no real modulus, i. e. both $\lambda^{\prime}$ and $\lambda^{\prime \prime}$ may be negative. The same applies to Poisson's ratio, but while limits can be set to the magnitudes of $v^{\prime}$ and $v^{\prime \prime}$, this is not so for $\lambda^{\prime}$ and $\lambda^{\prime \prime}$.

A useful proposition connecting the signs of $v^{\prime}$ and $v^{\prime \prime}$ on one hand and the sign of $\lambda^{\prime}$ on the other hand can be derived however.

From table 1 it is read:

$\lambda^{*}=\frac{3 \kappa^{*} v^{*}}{1+v^{*}}$,

from which it follows:

$\lambda^{\prime}=3 \kappa^{\prime} \frac{v^{\prime}+v^{\prime 2}+v^{\prime \prime 2}-v^{\prime \prime} \tan \delta_{\kappa}}{\left(1+v^{\prime}\right)^{2}+\left(v^{\prime \prime}\right)^{2}}$.

From eq. [36] it is concluded:

$\left.\begin{array}{c}v^{\prime}>0 \\ v^{\prime \prime}<0\end{array}\right\} \rightarrow \lambda^{\prime}>0$.

A similar proposition cannot be established for $\lambda^{\prime \prime}$. With respect to the position of $\tan \delta_{\lambda} \equiv\left|\lambda^{\prime \prime}\right| \lambda^{\prime} \mid$ in [15] and [22] resp. it will be shown:

$\left.\begin{array}{l}\nu^{\prime \prime} \gtrless 0 \\ \lambda^{\prime}>0 \\ \lambda^{\prime \prime}>0\end{array}\right\} \rightarrow \tan \delta_{\kappa} \lessgtr \tan \delta_{\lambda}$.

Eq. [35] can be written as

$$
\frac{\kappa^{*}}{\lambda^{*}}=\frac{1+v^{*}}{3 v^{*}} \text {. }
$$

Equating the imaginary parts of both sides of this equation we find:

$\frac{\kappa^{\prime} \lambda^{\prime}\left(\tan \delta_{\kappa}-\lambda^{\prime \prime} / \lambda^{\prime}\right)}{\left(\lambda^{\prime}\right)^{2}+\left(\lambda^{\prime \prime}\right)^{2}}=\frac{-v^{\prime \prime}}{3\left\{\left(v^{\prime}\right)^{2}+\left(v^{\prime \prime}\right)^{2}\right\}}$,

from which [38] is seen to be valid.

Eq. [38] was given before by Theocaris (5) in a different way where he took $\lambda^{\prime}>0$ and $\lambda^{\prime \prime}>0$ tacitly for granted, however. From [39] it also follows:

$\left.\begin{array}{rl}v^{\prime \prime} & \gtrless 0 \\ \lambda^{\prime} & <0 \\ \lambda^{\prime \prime} & <0\end{array}\right\} \rightarrow \tan \delta_{\kappa} \lessgtr \tan \delta_{\lambda}$.

For the remaining possibilities, viz. $\lambda^{\prime}>0$, $\lambda^{\prime \prime}<0$ and $\lambda^{\prime}<0, \hat{\lambda}^{\prime \prime}>0$, no bounds can be deduced.

Finally we prove:

$\left.\begin{array}{rl}v^{\prime} & >0 \\ v^{\prime \prime} & <0 \\ \lambda^{\prime \prime} & <0\end{array}\right\} \rightarrow \tan \delta_{v}>\tan \delta_{i}$.

From table 1 we read:

$\lambda^{*}=\frac{3 \kappa^{*} v^{*}}{1+v^{*}}$,

from which it is found:

$\frac{\lambda^{*}}{v^{*}}=3 \kappa^{*}-\lambda^{*}$.

So

$\operatorname{Im} \cdot \frac{\lambda^{*}}{v^{*}}=\frac{\lambda^{\prime} v^{\prime}\left(\tan \delta_{v}-\tan \delta_{\lambda}\right)}{\left(v^{\prime}\right)^{2}+\left(v^{\prime \prime}\right)^{2}}=3 \kappa^{\prime \prime}-\lambda^{\prime \prime}$,

from which, together with $[37],[41]$ is seen to be valid.

Taking into account the fact that there is a strong experimental evidence that $v^{\prime}>0\left(v^{\prime}>0.2\right.$ respectively) and $v^{\prime \prime}<0$ the main results can be summarized as follows:

$$
\left.\begin{array}{rl}
v^{\prime} & >0 \\
v^{\prime \prime} & <0
\end{array}\right\} \rightarrow \lambda^{\prime}>0,
$$

$$
\begin{aligned}
& \left.\begin{array}{rl}
v^{\prime} & >0 \\
v^{\prime \prime} & <0 \\
\lambda^{\prime \prime} & <0
\end{array}\right\} \rightarrow \tan \delta_{v}>\tan \delta_{\lambda}, \\
& \left.\begin{array}{l}
v^{\prime \prime}<0 \\
v^{\prime}>0
\end{array}\right\} \rightarrow \tan \delta_{G}>\tan \delta_{E}>\tan \delta_{\kappa}\left(>\tan \delta_{\lambda}\right) \\
& \left(\lambda^{\prime \prime}>0\right)
\end{aligned}
$$




$$
\left.\begin{array}{rl}
v^{\prime \prime} & <0 \\
v^{\prime} & >0.2 \\
\left(\lambda^{\prime \prime}>0\right)
\end{array}\right\} \rightarrow \tan \delta_{G}>\tan \delta_{E}>\tan \delta_{L}
$$

\section{Experimental evidence}

To check the relations deduced two illustrative examples will be given calculated from data published by the present author (3). In fig. 8 $v^{\prime}$ and $v^{\prime \prime}$ are plotted vs. temperature for an isotactic polypropylene. This figure shows that over the whole temperature region measured $v^{\prime}>0.2$ and $v^{\prime \prime}<0$. In fig. $9 \lambda^{\prime}$ and $\lambda^{\prime \prime}$ are plotted vs. temperature for the same polymer. It is

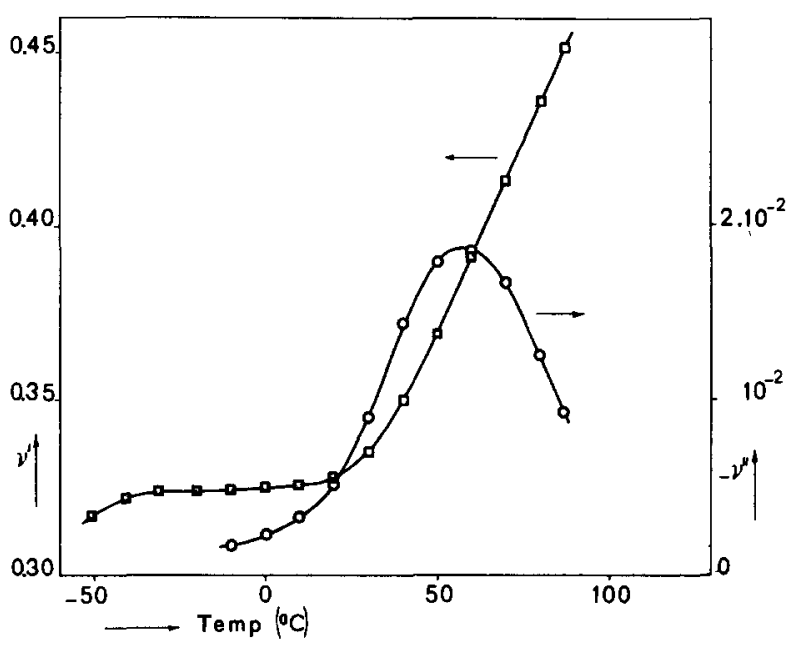

Fig. 8. Real part $v^{\prime}$ and imaginary part $v^{\prime \prime}$ of complex Poisson's ratio $v^{*}$ vs. temperature for an isotactic polypropylene. Frequency $5 \mathrm{MHz}$ seen that $\lambda^{\prime}>0$ in accordance with [37], while here $\lambda^{\prime \prime}>0$ too. In figs. 10 and 11 similar graphs are plotted for a high density polyethylene. Here too $v^{\prime}>0.2, v^{\prime \prime}<0$ and $\lambda^{\prime}>0$ over the whole temperature region, but now $\lambda^{\prime \prime}$ changes sign three times. So for the first polymer [44] applies throughout, while for the second one the last part of [44] $\left(\tan \delta_{\kappa}>\tan \delta_{\lambda}\right.$ with $\left.\tan \delta_{\lambda} \equiv \frac{\left|\lambda^{\prime \prime}\right|}{\lambda^{\prime}}\right)$ does not necessarily hold. In figs. 12 and 13 the relevant quantities are plotted for both polymers. (For clarity in fig. 13 $\lambda^{\prime \prime} / \lambda^{\prime}$ is plotted instead of $\tan \delta_{\lambda}$.) From these figures it is seen that [44] holds, whereas this is not the case for [23] as in some temperature regions $\tan \delta_{\kappa}>\tan \delta_{v}$, while in other ones $\tan \delta_{\kappa}<\tan \delta_{v}$. Moreover it is seen that the curves in fig. 13 are in accordance with [41].

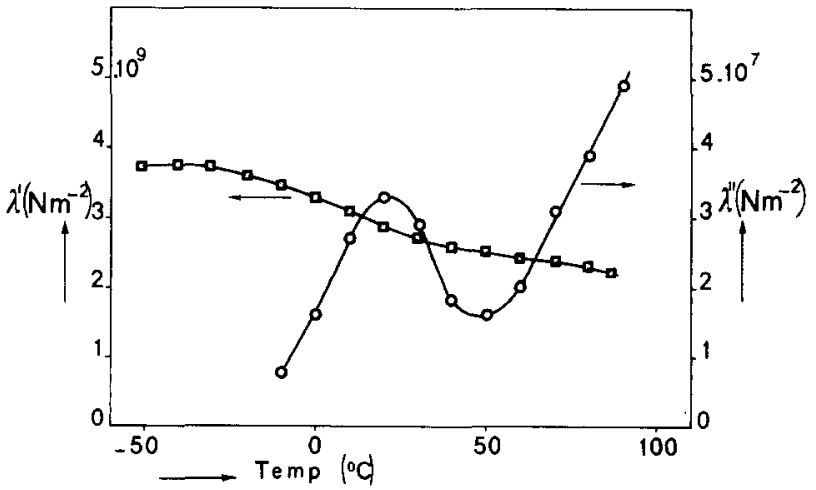

Fig. 9. Real part $\lambda^{\prime}$ and imaginary part $\lambda^{\prime \prime}$ of the complex second Lamé function vs. temperature. Same polymer as in fig. 8 and same frequency.

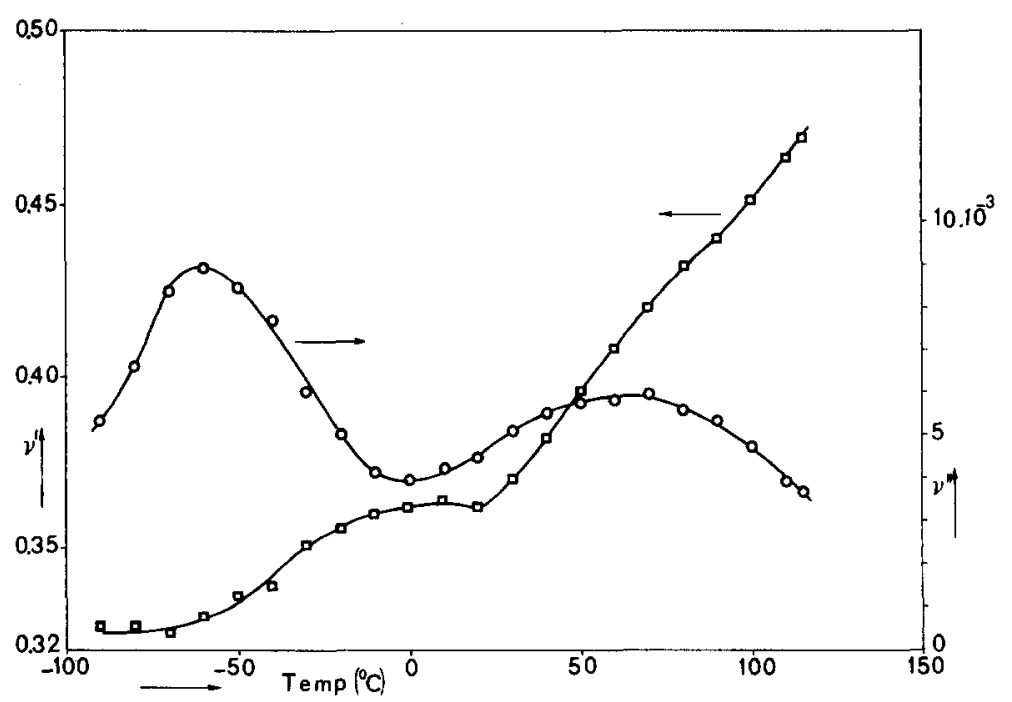

Fig. 10. Real part $v^{\prime}$ and imaginary part $v^{\prime \prime}$ of complex Poisson's ratio $v^{*}$ vs. temperature for a high density polyethylene. Frequency 


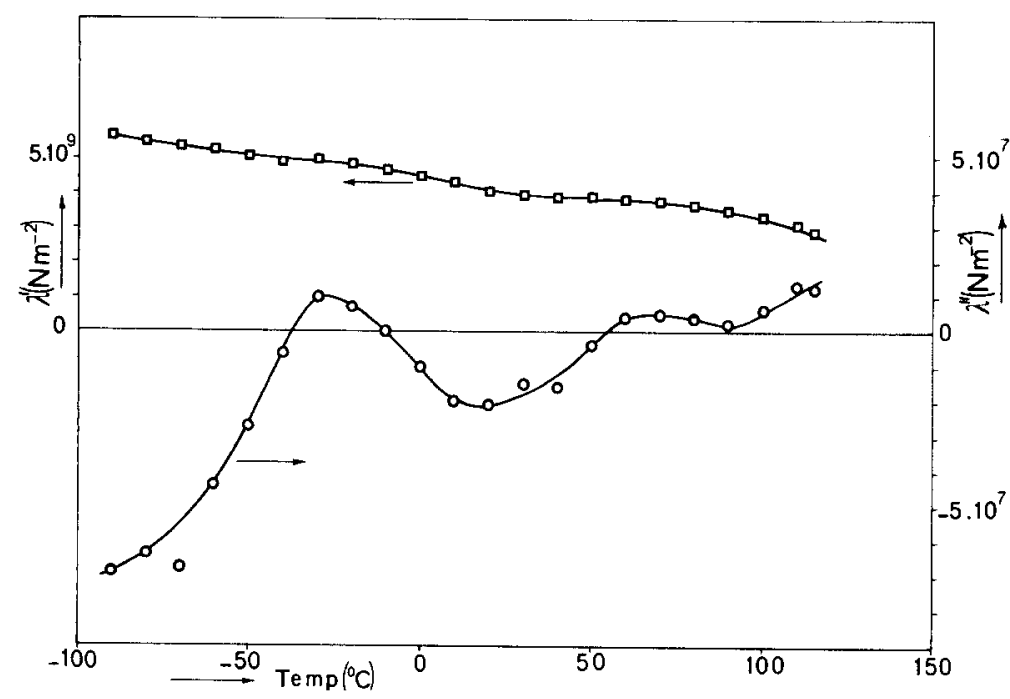

Fig. 11. Real part $\lambda^{\prime}$ and imaginary part $\lambda^{\prime \prime}$ of the complex second Lamé function vs. temperature. Same polymer as in fig. 10 and same frequency

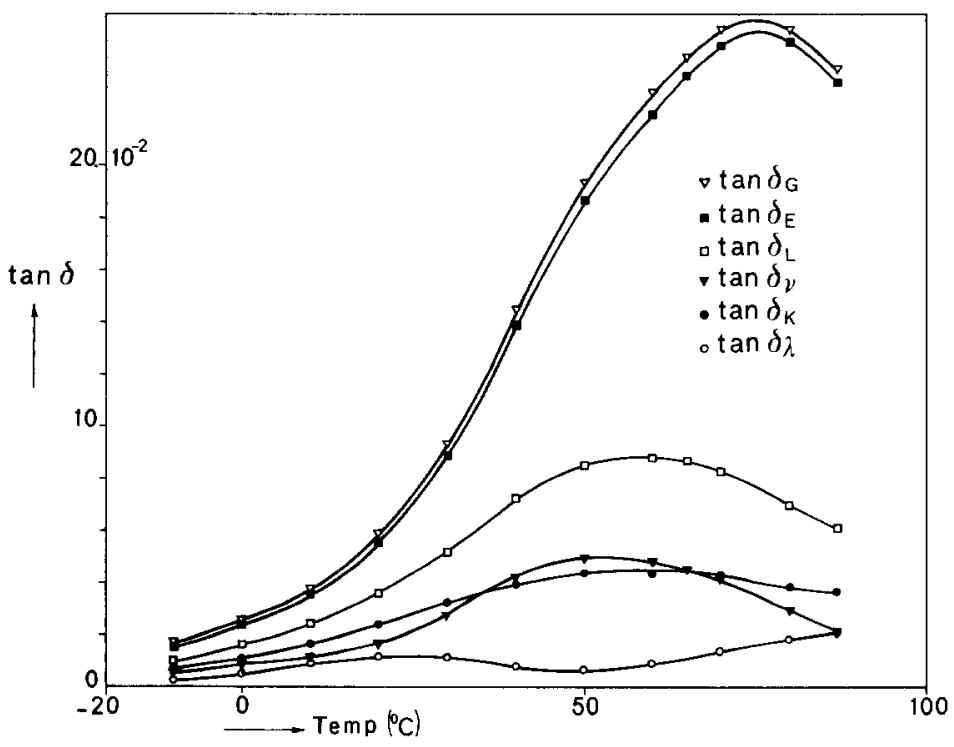

Fig. 12. Various loss tangents vs. temperature for the polymer of fig. 8 . Frequency $5 \mathrm{MHz}$

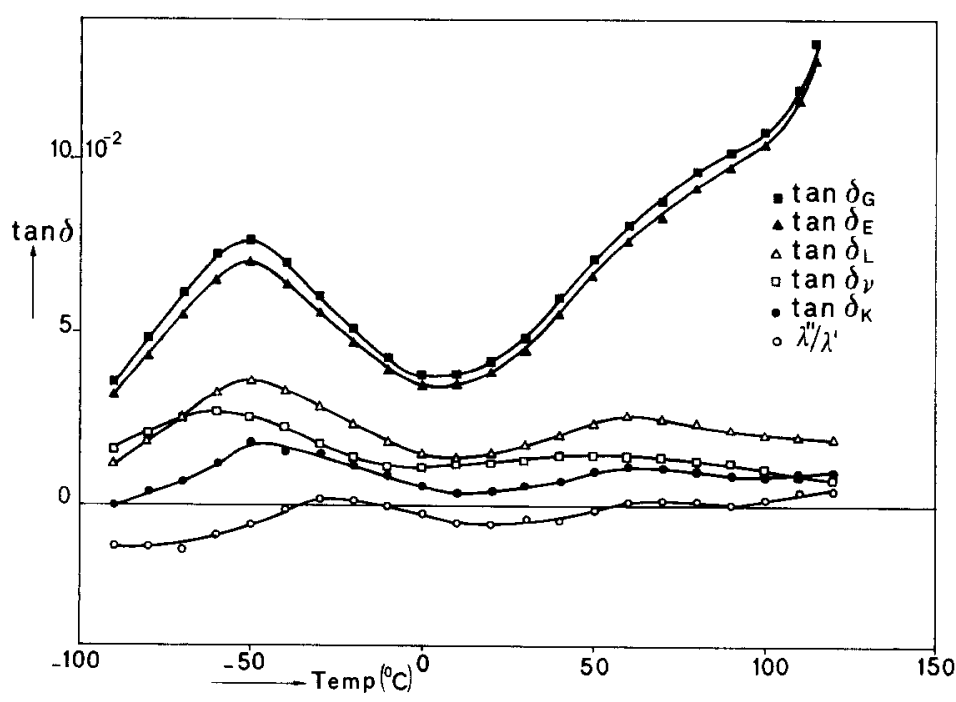

Fig. 13. Various loss tangents vs. temperature for the polymer of fig. 10 Frequency $5 \mathrm{MHz}$ 


\section{Phase shift in stress-strain relationship}

Let us consider an uniaxial stress experiment and let us face a cube oriented under $\pi / 4$ radians with the stress direction, see fig. 14. The cube will be sheared over the angle $\psi$ and it is readily shown that for small deformations the shear angle is given by $\psi=(1+v) \varepsilon$, which for harmonic stress turns into

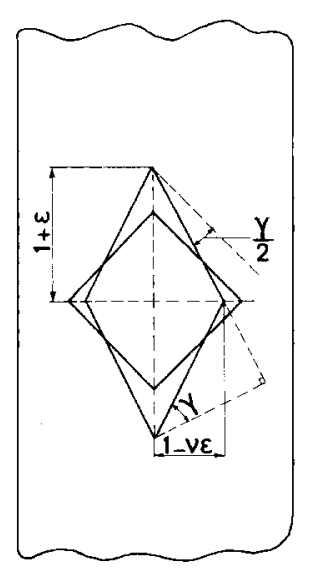

Fig. 14. Physical interpretation of $\alpha \equiv-\frac{v^{\prime \prime}}{1+v^{\prime}}$

$\psi=\left(1+. v^{*}\right) \varepsilon$.

From [45] and [5] we find that the principle strain $\varepsilon$ is in advance of $\psi$ by the angle

$\delta_{G}-\delta_{E}=\operatorname{arct} \alpha$.

The cube mentioned is subjected to the maximum shear stress in the sample, only, which amounts to $T_{11} / 2$. From this fact and eq. [4] the relationship [45] is also directly found. Eq. [45] provides in principle the possibility of measuring $\alpha$.

No such simple illustration of $\gamma$ could be found. A physical interpretation of $\gamma$ can be given in the following way, however. From table 1 it is seen that

$$
1-v^{*}=\frac{v^{*} L^{*}}{\lambda^{*}}
$$

Now when a longitudinal wave is propagating in a material a volume element only shows a strain in the direction of propagation, i.e. no lateral contraction occurs. The components of the stress tensor for this type of deformation are:

$T_{11}=T_{22}=\lambda^{*} \varepsilon, T_{33}=\left(\lambda^{*}+2 G^{*}\right) \varepsilon, T_{i \neq j}=0$.
The deformation therefore can be decomposed into two elementary deformations, viz. a universal expansion due to a hydrostatic pressure $\lambda^{*} \varepsilon$ and a uniaxial strain due to the stress $2 G^{*} \varepsilon$ in the direction of propagation. For the lateral contraction to be zero we must have

$\frac{\lambda^{*}}{3 \kappa^{*}}=\frac{2 G^{*} v^{*}}{E^{*}}$.

Further we see from table 1:

$\lambda^{*}=\frac{L^{*} v^{*}}{1-v^{*}}$.

By eliminating $\lambda^{*}$ from [47] and [48] eq. [14] can be found.

For the volume change $\Delta V / V$ in an experiment with a harmonic uniaxial strain we have:

$\Delta V / V=\left(1-2 v^{*}\right) \varepsilon$.

From [49] and [2] it follows that $\Delta V / V$ leads $\varepsilon$ by the angle $\delta_{E}-\delta_{\kappa}=\operatorname{arctg} \beta$.

In fig. 15 the various quantities for the isotactic polypropylene mentioned before are shown. From [49] we further see that for volume changes to be in phase with longitudinal

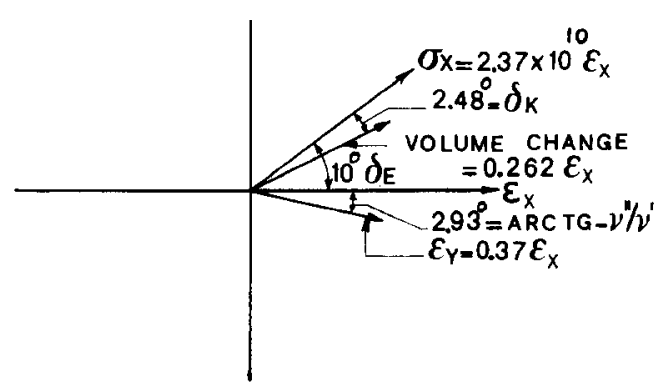

Fig. 15. Phase relationships for the deformations and volume change for the polymer of fig. 8. Frequency $5 \mathrm{MHz}$, temperature $50^{\circ} \mathrm{C}$

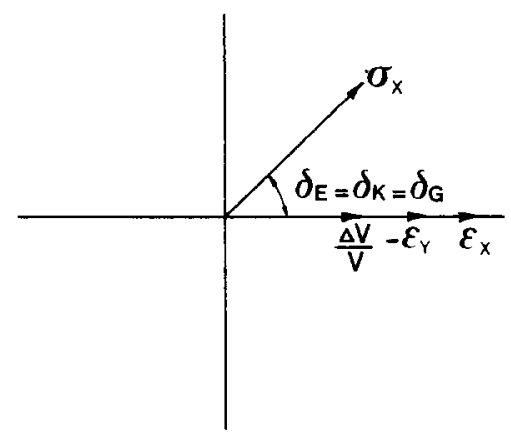

Fig. 16. Phase relationships assuming volume change to be in phase with longitudinal deformation 
deformations $v^{\prime \prime}$ must be zero, which is illustrated in fig. 16. Figures similar to our figs. 15 and 16, using the same experimental data, were given previously by Rigbi (8). Unfortunately this author (2) used two wrong arguments, viz.

i) for $v^{\prime \prime}=0$ volume changes are in phase with the principle stresses but at a phase angle $\delta$ in advance of the principle strains;

ii) if volume changes are assumed in phase with longitudinal deformations, $\kappa^{\prime \prime}=0$.

From [49] it is evident that both arguments are wrong and so are the figures of Rigbi (3) based on these arguments.

Finally fig. 16 suggests a new experimental method for determining the loss angle in compression, $\delta_{k}$, by measuring the angle which the volume change lags the stress.

\section{Discussion}

In the final relations [37], [40], [43] and [44] we assumed $v^{\prime}>0, v^{\prime \prime}<0$, respectively $v^{\prime}>0.2$, $v^{\prime \prime}<0$. Although theoretically $-1<v^{\prime}<\frac{1}{2}$ experimentally established data for $v^{\prime}$ are always positive*). Negative values of $v^{\prime}$ mean that in an extension experiment length- and lateral deformations have the same sign. Though formally allowed one would not expect such a behaviour, and in fact some authors (10) reject negative values of $v^{\prime}$ for that reason. Poisson (11) assumed in his pioneering work central forces and so arrived at a value of $v^{\prime}=0.25$, a value which appears to be the lowest one found experimentally. Measurements of several investigators on many polymers, including filled ones, so far always revealed $v^{\prime}>0.2$.

For the imaginary part of $v^{*}$ we have

$-\frac{3}{4} \leq v^{\prime \prime} \leq \frac{3}{4}$,

$v^{\prime \prime}>0$ would mean $\tan \delta_{\kappa}>\tan \delta_{G}$, which is not in agreement with extensive experimental evidence. Some authors quote positive values of $v^{\prime \prime}$ from their experiments, but these results are a matter of considerable doubt. So Thomson (12) not only found for an urethane rubber at room temperature and $100 \mathrm{~Hz} v^{\prime \prime}>0$, but also $v^{\prime} \sim 0.2$, a value even lower than found for diamond $\left(v^{\prime}=0.25\right)$. Measurements of Lepie (13) revealed a value of $v^{\prime \prime}=0.1$ (values of $v^{\prime \prime}$ in fig. 5 of his

*) An exception is reported by Lewis and Tobin (9). As these authors also find values of $v^{\prime}>\frac{1}{2}$ not too much weight should be attached to their results, however. paper show an error in calculation of a factor 2) at room temperature and $0.5 \mathrm{~Hz}$ for a black filled rubber. From his data we calculated in the limit of zero strain $\tan \delta_{G}=0.07$ and $\tan \delta_{\kappa}=1.7$, a set of data which is hard to believe. On the other hand measurements of the present author on several polymers $(3,4,14,15)$ always revealed $v^{\prime \prime}=0$.

Summarizing one can state that there is a strong experimental evidence that $v^{\prime}>0.2$ and $v^{\prime \prime}<0$ for polymers, but there is no theoretical base for this rule and it may break down for other materials and/or temperature respectively frequency regions.

\section{Summary}

Starting from the relations between complex dynamic moduli simple diagrams are deduced connecting the locus of complex Poisson's ratio $v^{*} \equiv v^{\prime}+i v^{\prime \prime}$ in the complex $v^{*}$ plane with differences between various loss angles. From these diagrams the sequence of magnitudes of several loss angles appearing in linear viscoelastic theory is deduced. Although theoretically this sequence depends on the values of $v^{\prime}$ and $v^{\prime \prime}$, it is found experimentally that for polymeric materials, due to the fact that the values of $v^{\prime}$ and $v^{\prime \prime}$ are constrained to limited ranges, general rules can be given. The sequences deduced are compared with experimental data. Finally some relations are used to illustrate the phase relationships between stress and deformations in an uniaxial stress experiment. From these relations a new method for measuring the loss angle in compression is suggested.

\section{Zusammenfassting}

Mit Hilfe der Beziehungen zwischen komplexen dynamischen Moduln wurden einfache Diagramme konstruiert, in denen der geometrische Ort des komplexen Poisson-Verhältnisses $v^{*} \equiv v^{\prime}+i v^{\prime \prime}$ in der komplexen $v^{*}$-Ebene mit Differenzen zwischen verschiedenen Verlustwinkeln verbunden werden. Aus diesen Diagrammen kann auf die Reihenfolge der Größe mehrerer in der linearen viskoelastischen Theorie auftretender Verlustwinkel gefolgert werden. Obwohl diese Reihenfolge theoretisch von den Werten von $v^{\prime}$ und $v^{\prime \prime}$ abhängig ist, wird experimentell gefunden, daß für polymere Substanzen allgemeine Regeln gegeben werden können. Dies findet seine Ursache in der Tatsache, daß die Werte von $v^{\prime}$ und $v^{\prime \prime}$ auf begrenzte Gebiete beschränkt sind. Zum Schluß werden für einen einachsigen Spannungsversuch die Phasenbeziehungen zwischen den Spannungen und den Verformungen illustriert. Mit Hilfe dieser Phasenbeziehungen wird eine neue Methode für die Messung des Verlustwinkels in Kompression vorgeschlagen.

\section{References}

1) Gottenberg, $W . G ., R . M$. Christensen, Trans. Soc. Rheol. 7, 171 (1963).

2) Rigbi, Z., Rheol. Acta 5, 28 (1966). 
3) Waterman, H. A., Kolloid-Z. u. Z. Polymere 192, 1 (1963).

4) Waterman, H. A., Kolloid-Z. u. Z. Polymere 192, 9 (1963).

5) Theocaris, P. S., Kolloid-Z. u. Z. Polymere 235 , 1182 (1969).

6) Christensen, R. M., Trans. Soc. Rheol. 16, 603 (1972).

7) Schwarzl, F. R., A. Burgers, Private communication.

8) Rigbi, Z., Appl. Pol. Symp. 5, 1 (1967).

9) Lewis, F. A., M. O. Tobin, Trans. Soc. Plast. Engrs. 1, 177 (1961).

10) Schwarzl, F. R., Kolloid-Z. 148, 47 (1956).

11) Poisson, S. D., Mémoire sur l'équilibre et le mouvement des corps élastiques, Paris, Mém. de l'Acad. t. 8 (1829).
12) Thomson, K. C., J. Appl. Polymer Sci. 10, 1133 (1966).

13) Lepie, A. H., Rev. Sci. Instrum. 40, 1004 (1969).

14) Heijboer, J., L. C. E. Struik, H. A. Waterman, M. P. van Duijkeren, J. Macromol. Sci., Phys. B 5, 375 (1971).

15) Waterman, H. A., L. C. E. Struik, J. Heijboer, M. P. van Duijkeren, In: R. W. Douglas and B. Ellis (Eds.), Amorphous Materials, p. 29 (New York 1972).

Author's address :

H. A. Waterman

Department of Applied Physics

Twente University of Technology

Enschede (The Netherlands) 